\title{
Impedance Control of Redundant Manipulators for Safe Human-Robot Collaboration
}

\author{
Fanny Ficuciello, Luigi Villani, Bruno Siciliano \\ Dipartimento di Ingegneria Elettrica e Tecnologie dell'Informazione, \\ Università di Napoli Federico II, Napoli, Italy, \\ \{fanny.ficuciello,luigi.villani,bruno.siciliano\}@unina.it
}

In this paper, the impedance control paradigm is used to design control algorithms for safe human-robot collaboration. In particular, the problem of controlling a redundant robot manipulator in task space, while guaranteeing a compliant behavior for the redundant degrees of freedom, is considered first. The proposed approach allows safe and dependable reaction of the robot during deliberate or accidental physical interaction with a human or the environment, thanks to null-space impedance control. Moreover, the case of control for comanipulation is considered. In particular, the role of the kinematic redundancy and that of the impedance parameters modulation are investigated. The algorithms are verified through experiments on a $7 R K U K A$ lightweight robot arm.

Keywords: robot manipulators; physical human-robot interaction; impedance control

\section{Introduction}

The need of safe and dependable robots operating in the close vicinity to humans or directly interacting with persons is growing in a wide range of application domains, ranging from domestic environments to industry. This requires a major technology shift from classical industrial robots, which are closed in cages to guarantee safety, to a new generation of robots suitable to be used in close collaboration with humans.

These robots must be designed with a high degree of compliance to reduce the interaction forces, both in the case of collision and during physical collaboration with humans. Moreover, a safe human-robot coexistence must be guaranteed combining different control strategies.

Collisions should be avoided using exteroceptive sensors, such as video cameras or depth sensors, together with fast collision avoidance control algorithms. Also, appropriate collision detection and reaction strategies must be adopted in case of collisions that cannot be avoided. The reaction strategies are aimed at immediately removing the robot from the collision area. Nevertheless, in the case of redundant robots, it is 
possible to preserve as much as possible the execution of the end effector task by projecting the reaction torques into the null space of the main task.

Similarly, in the case of accidental or intentional physical interaction of a human with the robot's body, a suitable compliance control strategy can be adopted for the redundant degrees of freedom of the robot, which possibly does not interfere with the task assigned to the robot's end effector. The resulting approach, proposed in the first section of this paper, can be denoted as task space control with null space compliance.

On the other hand, a number of robotic tasks require intentional physical interaction of humans with robots. This happens, for example, in co-manipulation tasks, where the human guides the robot's end effector. For these tasks, suitable control strategies ensuring the robot's compliance at the end effector must be adopted and kinematic redundancy can be exploited to enhance intuitiveness and stability of the physical human-robot interaction. This problem is addressed in the second section of the paper.

The paradigm common to the two approaches is the use of impedance control [1] for human-robot physical interaction. They have been considered separately in the references [2], for what concerns null-space impedance control, and [3] for variable impedance control at the end effector.

\section{Null-Space Impedance Control for Human-Robot Co- existence}

When a robot works close to humans, interaction may occurs with the robot's body. Safety can be guaranteed by ensuring a compliant behaviour to the robot's joint. In the presence of kinematic redundancy, the compliant behaviour can be projected in the null space of the main task, so that the latter can be correctly executed. In other words, the control goal is to minimize the error of the main task and at the same time, to ensure safe interaction through active compliance in the null space of the main task.

Active compliance can be obtained using impedance control, which has been extensively studied in the literature. The compliant behavior usually is realized in the task space to control the interaction of the end effector. However, an impedance behavior can be imposed also in the null space of the main task to ensure safety.

To this purpose, two control schemes which do not require direct joint torque measurements are presented. The first scheme is based on a disturbance observer which estimates the external forces acting on the task variables on the basis of the task space error. The second scheme relies on the momentum-based observer [4]. In both cases, the overall stability of the system, with asymptotic convergence of the main task and a desired impedance behavior in the null space of the main task, can be proven. More details and the proofs are in [2]. 


\subsection{Null-Space Impedance Control}

The dynamic model of a $n$-link robot manipulator can be expressed by

$M(q) \ddot{q}+C(q, \dot{q}) \dot{q}+g(q)+\tau_{\text {ext }}=\tau$,

with standard notation. In this model, $\tau$ is the input vector torque while $\tau_{\text {ext }}$ is the torque resulting from external interaction. The well-known model-based resolved acceleration control can be adopted to compute the driving torques

$\tau=M(q) \ddot{q}_{c}+C(q, \dot{q}) \dot{q}+g(q)$,

where $\ddot{q}_{c}$ is the joint command acceleration to be suitably designed.

For a redundant manipulator, redundancy lets us to have some kind of joint impedance and task space control simultaneously. The so-called null-space impedance can be realized in the null space of the main task to control the interaction on the robot's body. The corresponding command joint acceleration in (2) is given by

$\ddot{q}_{c}=J^{\dagger}\left(\ddot{x}_{c}-\dot{J} \dot{q}\right)+N\left(\ddot{q}_{d}+M_{d}^{-1}\left(B_{d} \dot{\tilde{q}}+K_{d} \tilde{q}\right)\right)$,

which produces the task space and null-space closed-loop behavior respectively as follows

$\ddot{x}_{c}-\ddot{x}=J M^{-1} \tau_{\text {ext }}$,

$N\left(\ddot{\tilde{q}}+M_{d}^{-1}\left(B_{d} \dot{\tilde{q}}+K_{d} \tilde{q}\right)-M^{-1} \tau_{\text {ext }}\right)=0$.

Here $\ddot{x}_{c}$ is the command acceleration in the task space, $\tilde{q}=q_{d}-q$ where $q_{d}$ is the desired trajectory or a rest configuration in the joint space, $J^{\dagger}$ is any (weighted) right pseudo-inverse of the task space Jacobian matrix $J, N=\left(I-J^{\dagger} J\right)$ is the null-space projection matrix, and $M_{d}, B_{d}$ and $K_{d}$ are the impedance matrices. This choice of $\ddot{q}_{c}$ allows the joint space impedance in the null-space of the main task to be realized, provided that the desired inertia matrix is chosen as $M_{d}=M(q)$. On the other hand, the main task experiences errors as a result of the external torques that are applied on the robot's body. It can be easily shown that an arbitrary $M_{d}$ can be set only if the measurement of $\tau_{\text {ext }}$ is available to the controller; in this case, also the influence of $\tau_{\text {ext }}$ on the main task can be cancelled out.

\subsection{Task-Based Observer}

The following theorem is given for correct execution of the main task, while ensuring a compliant behaviour of the robot's body.

Theorem 1 . Let us denote with $\hat{\tau}$ the estimated external torque and with $\tilde{\tau}=\tau_{\text {ext }}-\hat{\tau}$ the estimation error. Also, define the error $s=\dot{\tilde{x}}+P \tilde{x}$, where $P$ is a positive definite diagonal matrix and $\tilde{x}=x_{d}-x$. Then, for selected constant diagonal positive definite matrix $K$ and constant positive definite matrix $\Gamma_{f}$, the control law

$$
\begin{aligned}
\tau & =J^{T} \Lambda\left(\ddot{x}_{d}+P \dot{\tilde{x}}-\dot{J} \dot{q}\right)+J^{T}\left(\frac{1}{2} \dot{\Lambda}+K\right)-J^{T} J^{\# T} \hat{\tau} \\
& +M N_{\#}\left(\ddot{q}_{d}+M^{-1}\left(B_{d} \dot{\tilde{q}}+K_{d} \tilde{q}\right)\right)+C(q, \dot{q}) \dot{q}+g(q),
\end{aligned}
$$


with the disturbance observer

$\dot{\tilde{\tau}}=-\Gamma_{f}^{T} J^{\#} s$,

guarantee that the $\tilde{x}$ and $\dot{\tilde{x}}$ go to zero asymptotically while the null-space impedance behavior is imposed. Moreover, the estimated disturbance remains bounded and the closed-loop system is stable.

In (5), $\Lambda=\left(J M^{-1} J^{T}\right)^{-1}$ is task inertia matrix, $J^{\#}=M^{-1} J^{T} \Lambda$ is the dynamically consistent generalized inverse [5] and $N_{\#}=\left(I-J^{\#} J\right)$.

It can be easily shown that the closed-loop dynamics for the null-space is

$N_{\#}\left(\ddot{\tilde{q}}+M^{-1}\left(B_{d} \dot{\tilde{q}}+K_{d} \tilde{q}\right)-M^{-1} \tau_{\text {ext }}\right)=0$,

corresponding to an impedance behavior in the null-space of the main task.

\subsection{Momentum-Based Observer}

Another method to ensure the correct execution of the main task during interaction is based on the collision detection algorithm presented by [4]. The basic concept is the computation of the $n$-dimensional residual vector

$r(t)=K_{I}\left(p(t)-\int_{0}^{t}\left(\tau+C^{T}(q, \dot{q}) \dot{q}-g(q)+r(\sigma)\right) d \sigma\right)$,

where $p(t)=M(q) \dot{q}$ is the robot generalized momentum and $K_{I}$ is a positive definite diagonal matrix. This vector can be computed using the measured signals $q$ and $\dot{q}$, and the commanded torque $\tau$, with initial conditions $r(0)=0$ and $p(0)=0$. The dynamics of $r$ is

$\dot{r}=-K_{I} r-K_{I} \tau_{\text {ext }}$,

corresponding to a filtered version of the real external torques, i.e. $r(t)=\tau_{\text {ext }}$. In the absence of interaction, assuming no noise and unmodeled disturbances, $r(t)=0$. As soon as interaction occurs, the components of $r$ will raise exponentially and will reach to the value of $-\tau_{\text {ext }}$.

A control algorithm similar to the one that was given by (5), using $r$ in place of $\hat{\tau}$, is adopted

$$
\begin{aligned}
\tau & =J^{T} \Lambda\left(\ddot{x}_{d}+K_{D} \dot{\tilde{x}}+K_{p} \tilde{x}-\dot{J} \dot{q}\right)-J^{T} J^{\# T} r \\
& +M N_{\#}\left[\ddot{q}_{d}+M_{d}^{-1}\left(B_{d} \dot{\tilde{q}}+K_{d} \tilde{q}\right)\right]+C(q, \dot{q}) \dot{q}+g(q),
\end{aligned}
$$

By defining the estimation error $\tilde{r}=r+\tau_{\text {ext }}$, the closed-loop task dynamics is

$\ddot{\tilde{x}}+K_{D} \dot{\tilde{x}}+K_{p} \tilde{x}=J M^{-1} \tilde{r}$

$\dot{\tilde{r}}=-K_{I} \tilde{r}+\dot{\tau}_{\text {ext }} . \quad$,

while the closed-loop dynamics for the null-space is the same as in (7). From the stability properties of the cascade systems it can be shown that when $\left\|\dot{\tau}_{\text {ext }}\right\|$ is bounded, $\tilde{x}$ is also bounded and specifically when $\dot{\tau}_{e x t}=0$ the system is asymptotically stable and $\tilde{x}, \dot{\tilde{x}} \rightarrow 0$. 


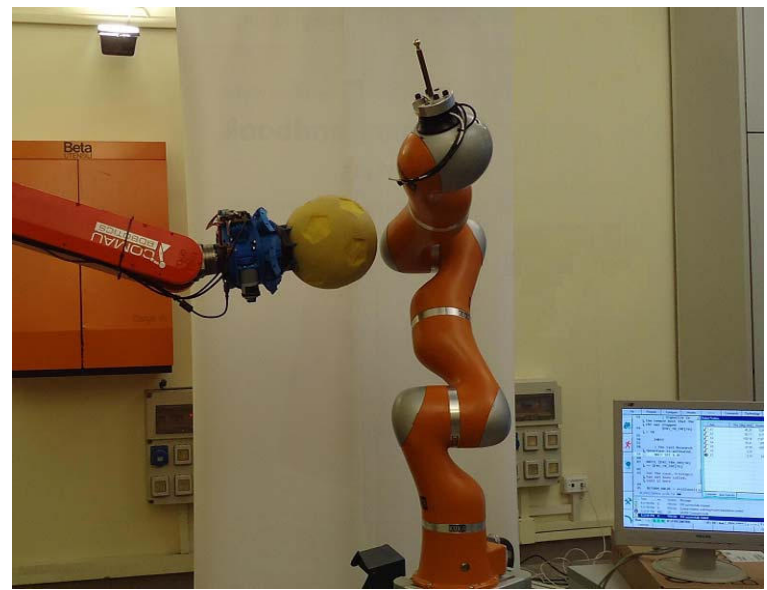

Figure 1

Elastic ball in interaction with a KUKA LWR4 arm

\subsection{Experimental Results}

The proposed approaches are verified experimentally on a 7 DOF KUKA LWR4 lightweight arm $(n=7)$. Control algorithms are executed through Fast Research Interface (FRI) library with a sampling rate of $2 \mathrm{~ms}$.

The experiments are performed for three cases: without observer, with task error based observer and with momentum based observer. In all the cases the position of the end effector is assumed as the main task $(m=3)$. Therefore, the robot has 4 degrees of redundancy $(r=4)$.

A constant configuration $q_{d}=[\pi / 4,-\pi / 6,0,-\pi / 1.8, \pi / 6,-\pi / 4,0]$ is considered, corresponding to the constant desired position of the end effector in the task space $x_{d}=[-0.242,-0.133,0.968]^{T}$.

The interaction occurs with an elastic ball of $1200 \mathrm{~N} / \mathrm{m}$ approximate stiffness at a point of the robot arm close to the fourth joint. While the end effector is commanded to be in the desired position, the sphere comes in contact with the robot, stops for $10 \mathrm{~s}$ and finally goes back far from the robot. In order to have the same scenario in all the experiments and guarantee repeatability, the ball is moved by a position controlled industrial robot with constant speed of $4.5 \mathrm{~cm} / \mathrm{s}$ along a straight line. A snapshot of the experimental setup is depicted in Fig. 1.

Case I, Interaction control without external interaction observer: The command acceleration given by (3) was considered with the gains $K_{p}=2000 I, K_{v}=90 I$, $M_{d}=M(q), B_{d}=0.4 I$, and $K_{d}=8 I$.

The corresponding main task error and the estimated external torques, obtained by the torque sensors available on LWR4 robot, together with the joints position, are shown in Fig. 2. The time interval when interaction occurs is identified by the two vertical lines. It can be observed that the task space error components are zero initially but, after the collision with the sphere, they increase and reach constant 

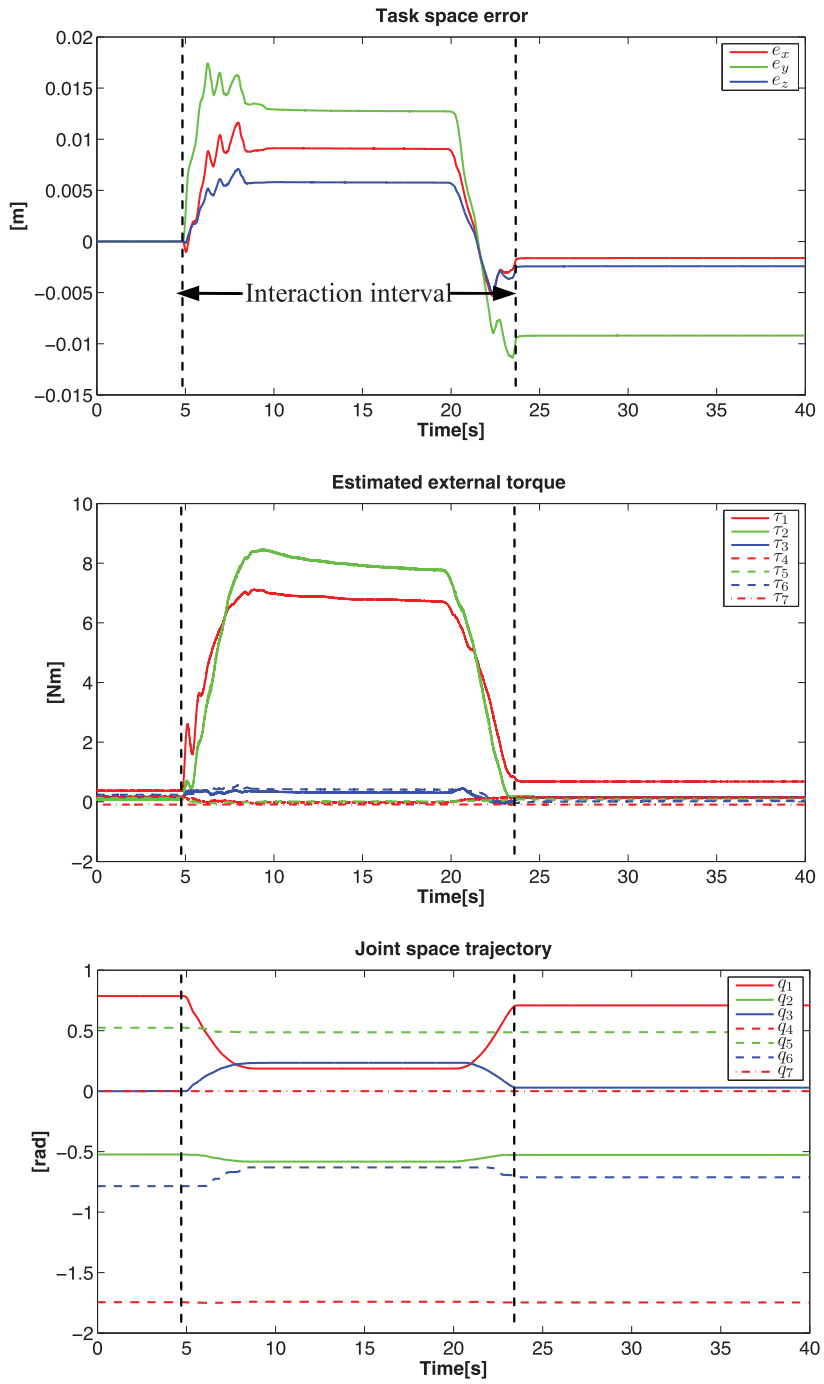

Figure 2

Task errors, external torques estimation evaluated from torque sensors and joint space trajectory, without observer

values when the sphere stops. When the sphere is retreated and contact is lost, the task error components become small but non-null, due to the presence of non negligible joint friction.

From the time histories of the joint variables in Fig. 2 it can be argued that, during the interaction, the configuration of the robot changes and the redundancy allows the manipulator to comply with the external forces. As soon as the contact is lost, the robot comes back to its desired configuration. The behavior of the arm in the null space can be set by properly choosing the control gains.

Case II, Interaction control with task error based disturbance observer: The previous experiment is repeated by using the control law (5) with disturbance observer 

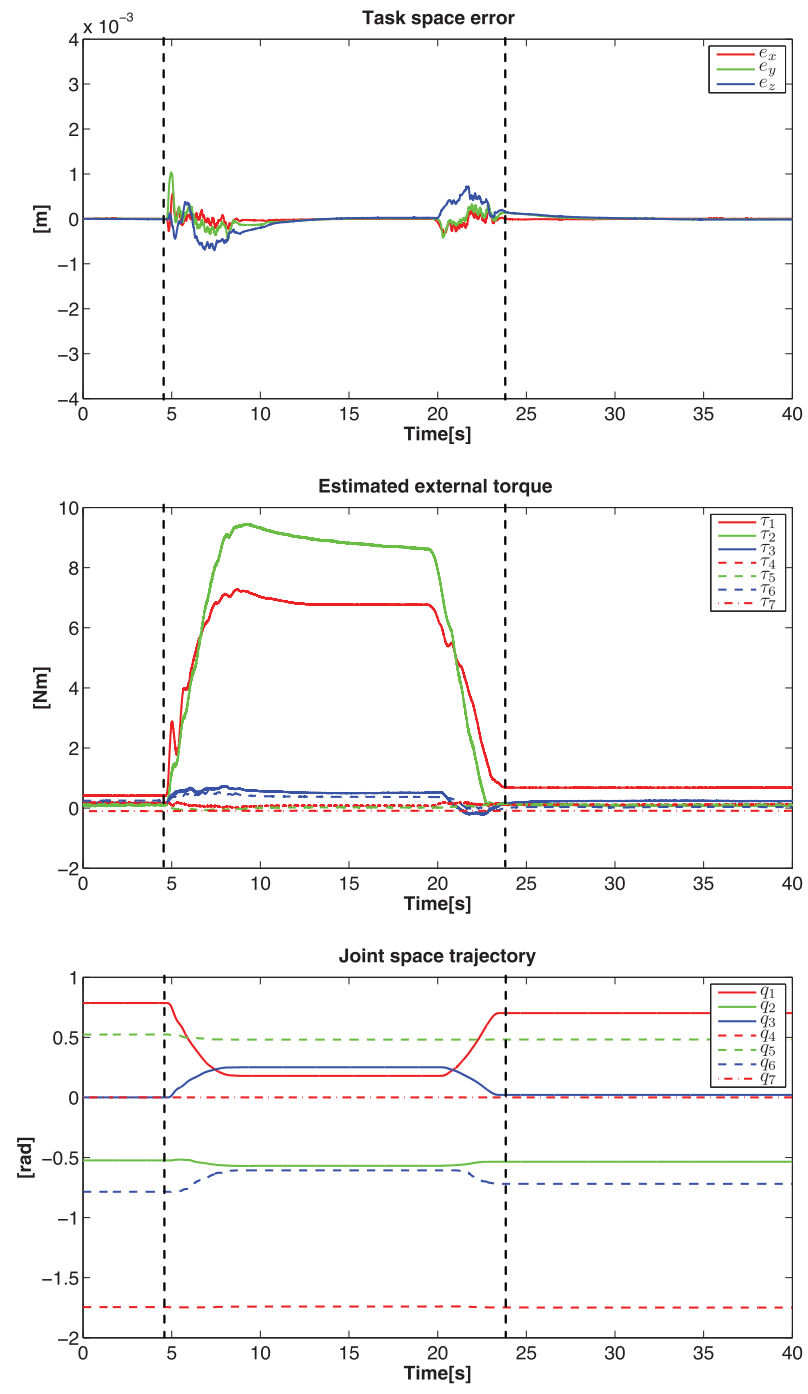

Figure 3

Task errors, external torques estimation evaluated from torque sensors and joint space trajectory, using task error based observer

(6). The parameters of the controller are tuned as $P=25 I, K=80 I$, and $\Gamma_{f}=0.125 I$ and the impedance matrices are selected as in the previous case.

The performance of the controller is shown in Fig. 3. Even though the external torque $\tau_{\text {ext }}$ is not constant during the first and the third phase of the interaction, namely when the sphere is approached and retreated, the controller performs very well and the task errors are more than three times lower than the previous case and the resulting interaction torques remain bounded. In the second phase of the interaction, when the sphere is at rest and a constant torque is applied, the task error converges to zero.

Comparing the plots of the time histories of the external torques and of the joint 

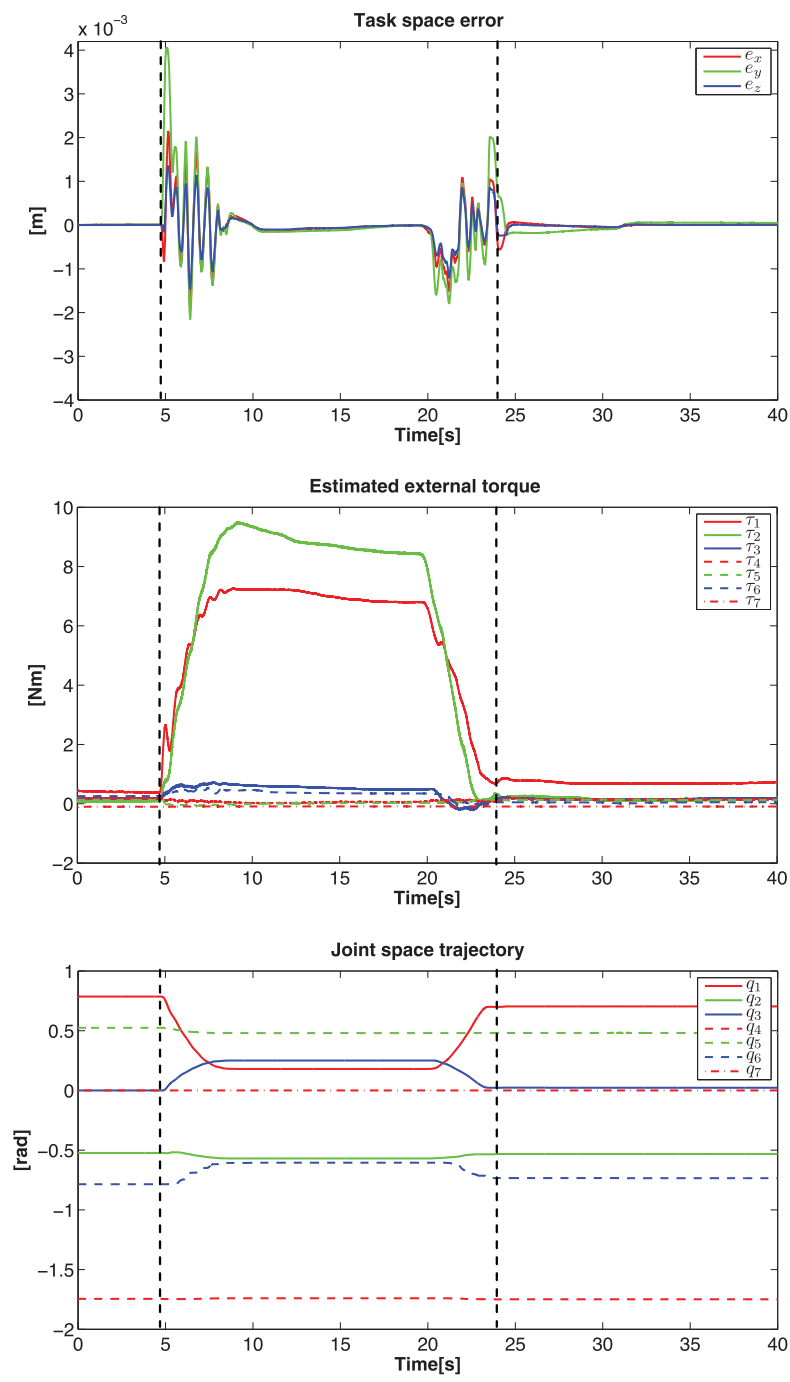

Figure 4

Task errors, external torques estimation evaluated from torque sensors and joint space trajectory, using momentum based observer

positions in Fig. 2 and Fig. 3, it can be inferred that the behavior of the robot in the null space does not change appreciably. Moreover, comparing the task space errors of Fig. 3 with those reported in Fig. 2, it can be observed that the control scheme with task error based observer not only reduces the error during the interaction, but allows also reducing the effects of friction, so that the task space error goes to zero when the contact is lost.

Case III, Interaction control with momentum based observer: The experiment is repeated using the momentum based observer (8) with $K_{I}=8 I$ and the control torque (10). The control gains and the impedance parameters are set as in Case I. The results are depicted in Fig. 4. It can be seen that the controller works very well during the constant phase of the interaction. However, when the external torque is 
is not constant, during the first and the third phase of the interaction, the task space error shows high frequency oscillations.

\section{Human-Robot Co-Manipulation}

Robot manual guidance and co-manipulation tasks are of great interest in both the service and industrial fields where physical human-robot interaction constitutes an added value for the realization and simplification of many applications, such as teaching by demonstration and robot-aided manufacturing. It is widely known in literature that impedance/admittance strategies are the most effective and natural way to handle physical interaction in the face of the unpredictability of human behaviours. Indeed, impedance control is an essential paradigm to ensure reliability and safety.

To enhance intuitiveness and stability in physical human-robot interaction during co-manipulation tasks both redundancy and impedance parameters modulation play an important rule. Through an extensive experimental study on a 7-DOF KUKA LWR4 arm, we show that variable impedance is more performant with respect to constant impedance and that redundancy resolution influences not only stability but also performance. For this purpose, an impedance strategy to control a redundant manipulator is defined in the Cartesian space and different modulation laws for the impedance parameters are tested in combination with different strategies to solve redundancy.

In order to make the end effector able to follow and adapt to the force exerted by the operator at the tip, the end effector dynamics can be set as a mass-damper system of equation

$\Lambda_{d} \ddot{x}+D_{d} \dot{x}=F_{\text {ext }}$,

where $\Lambda_{d}$ and $D_{d}$ are suitable inertia and damping matrices, that are positive definite and are usually set as constant diagonal matrices.

In detail, a suitably selected variable impedance strategy [7] has been compared to constant impedance. Among possible redundancy resolution criteria, two secondary task functions inspired to the dynamic conditioning index (DCI) [13], and to the kinematic manipulability index were selected and compared [6]. For the evaluation of the results, a writing task on a horizontal plane operated by a human has been selected as a case study. In order to have significant redundant degrees of freedom that can be used for the secondary task, the orientation was not considered.

A snapshot of the co-manipulation task is reported in Fig. 5, where the operator guides a paint marker mounted on the robot's tip along a path drawn on a paper sheet.

Fig. 6 summarizes the results which will be further discussed and detailed below. The performance level increases from left to right. It can be seen that the best solution is achieved using variable damping and secondary task functions inspired to the dynamic conditioning index. 


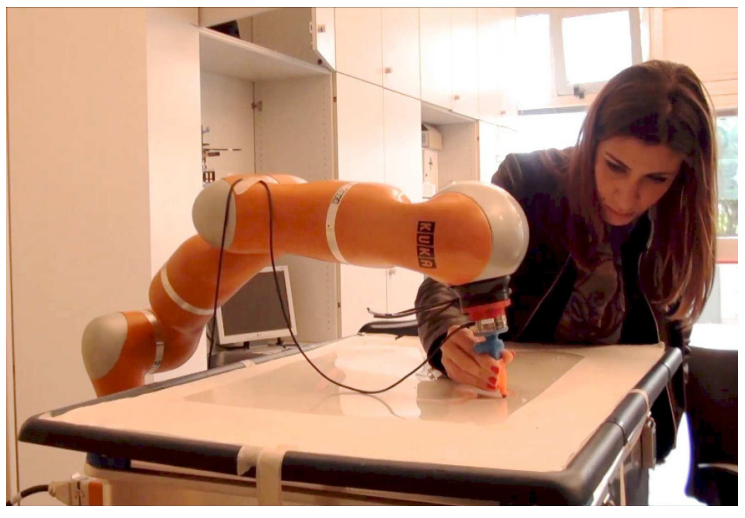

Figure 5

Snapshot of the co-manipulation task.

\begin{tabular}{|c|c|c|c|}
\hline \multicolumn{4}{|c|}{ Performance $(\mathrm{H}, \mathrm{e})$} \\
\hline MAN & $\mathrm{DCl}$ & MAN & $\mathrm{DCl}$ \\
\hline \multicolumn{2}{|c|}{ CONST IMP } & \multicolumn{2}{|c|}{ VAR IMP } \\
\hline
\end{tabular}

Figure 6

The results obtained with different combination of redundancy resolution and impedance strategies presented on the basis of the performance level in increasing order from left to right.

\subsection{Stability Issues}

Studies available in literature demonstrate that the stability of human-robot interaction, depends on the coupled dynamics of both interacting systems [12], which cannot be accurately modelled and evaluated as it involves also the estimation of human arm impedance, it depends also on the hardware namely, the robot kinematics and dynamics, the kind of transmission, the presence of friction and of structural compliance, and the kind of sensors and actuators $[10,9,11]$. Thus, in this work an experimental procedure has been set up to find the allowed range of variation of the impedance parameters where stability is preserved. In this section, only the results of the experimental procedure are reported in Fig. 7, while the whole procedure can be found in $[3,7]$. The same damping and the same mass has been set along all the directions of the Cartesian space, i.e., $D_{d}=D I$ and $\Lambda_{d}=\Lambda I$, with $\Lambda=\alpha \Lambda^{-}$, being ${ }^{-} \Lambda=4.2456 \mathrm{~kg}$ the maximum eigenvalue of the end effector inertia in a chosen initial configuration, and $0<\alpha \leq 1$ a scaling factor. The stability region for the parameters $D$ and $\alpha$ is that included between the continuous and the dotted line.

The stability region has been evaluated experimentally by setting a value of damping $D$ in the interval $[5,60] \mathrm{Ns} / \mathrm{m}$ and reducing the value of $\alpha$, starting from $\alpha=1$, until vibrations can be felt by an operator shaking the end effector in a neighborhood 
of an initial configuration. This configuration is chosen in such a way to have the effective Cartesian inertia diagonal with asymmetric distribution, i.e. one of the eigenvalues of the inertia matrix assumes a value $\bar{\Lambda}$ much bigger with respect to the others. Hence, the worst-case configuration for scaling (reducing) the end effector inertia is considered.

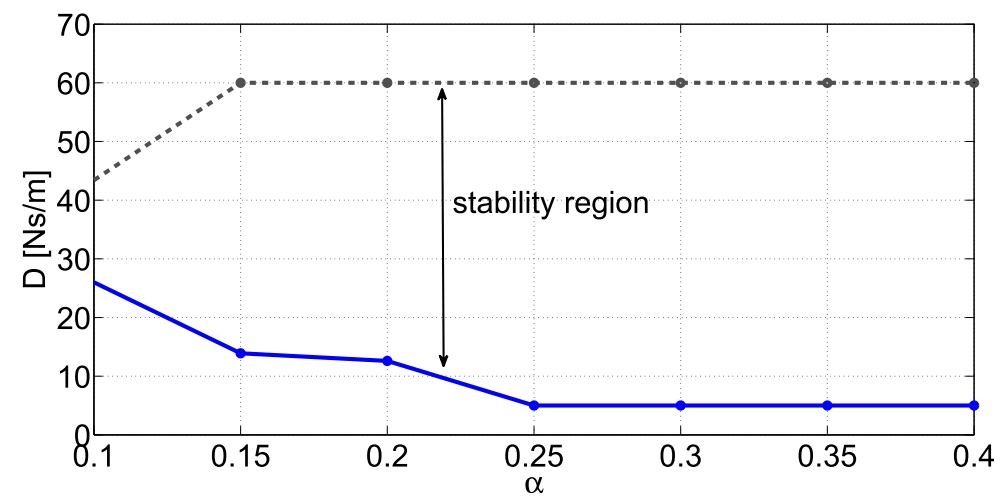

Figure 7

Range of minimum and maximum allowed damping $D$ for a given scaling factor $\alpha$ of the inertia matrix.

\subsection{Redundancy Resolution}

The experimental studies on a redundant robot demonstrate that redundancy has an important role on both stability and performance during human-robot physical interaction. Different strategies to solve redundancy have been applied and the results compared in terms of performances and in terms of stability. The maximization of the kinematic manipulability index [6] and the minimization of the DC index [13] are the two secondary tasks that have been tested. In Fig. 8 the time histories of DC index are reported in the two cases. In the top figure, that is the case where in the impedance equation low virtual inertia has been settled at the boundary of stability region, the task can be completed only if redundancy is used to decouple the effective Cartesian inertia at the end effector of the robot. Otherwise, when manipulability index is exploited, the task is interrupted because instability occurs. In the bottom figure the virtual inertia is settled high thus the stability is always preserved. This happened since instability is likely to occur during interaction when the controller attempts to impose to the robot impedance dynamics that differs signicantly from the intrinsic hardware dynamics.

About performances related to redundancy resolution, the methods have been compared using two different impedance laws, one with constant parameters (set as $\Lambda=1.1 \mathrm{~kg}, D=60 \mathrm{Ns} / \mathrm{m}$ ) and one with variable damping (low constant mass, $\Lambda=1.1 \mathrm{~kg}$ ). Since the assigned task consists in pursuing a given path, a significant measure of performance is the error between the reference and the actual path, that can be defined in different ways. A very simple measure is the absolute value of the difference between the length of the path drawn in cooperation with the robot, 

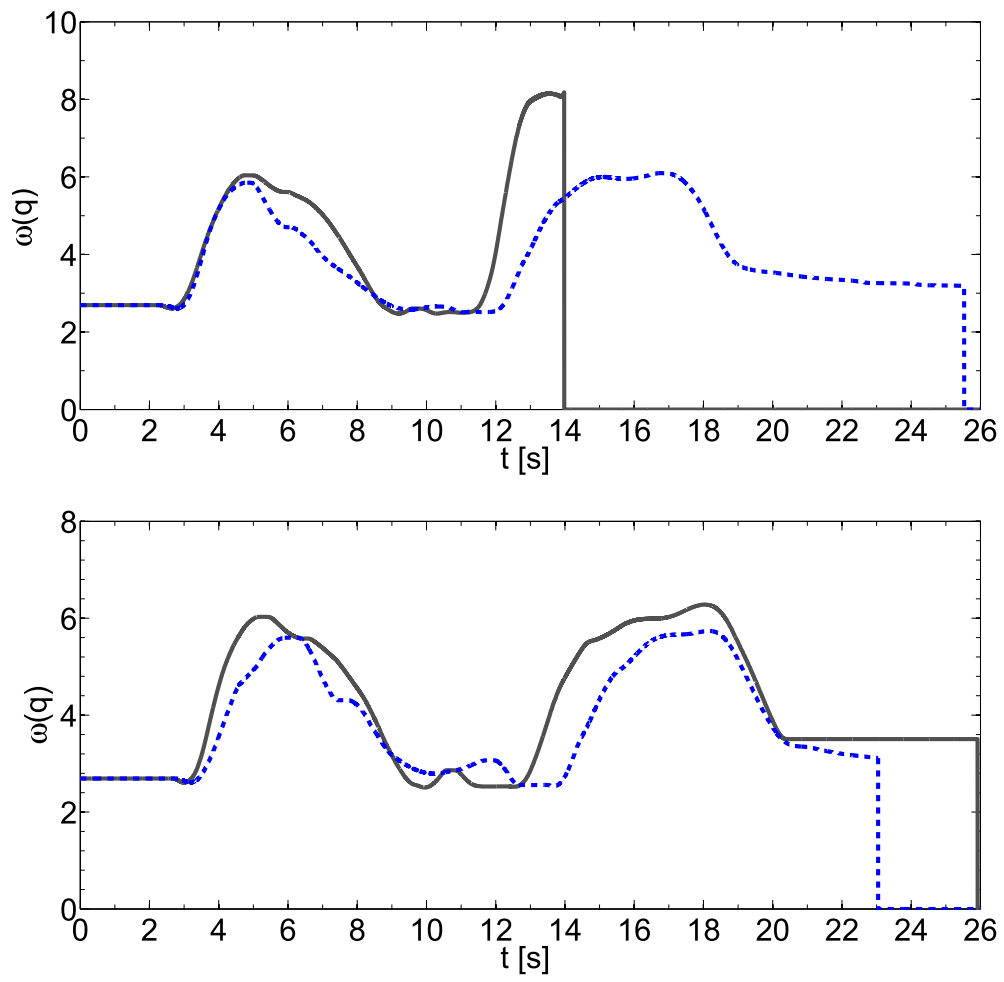

Figure 8

Time histories of the values of DC index in the case of low (top) and high (bottom) virtual inertia. The continuous lines represent the DC index when redundancy is used to increase manipulability. The dashed lines represent the DC index when redundancy is used to minimize the DC index.

$l_{e}$, and the ideal path length, $l_{d}$, namely the length error:

$e=\left|l_{d}-l_{e}\right|$.

Another performance parameter is the execution time $H$ of the trajectory, defined as the difference between the time when the entire path is completed and the time when the drawing tool touches the paper on the desk to start writing. In order to obtain quantities that overcome the skills of the singular operator, the above parameters are evaluated as the average on the performance of more subjects.

The results of the tests are reported in Fig. 9, where the error on the length of path $e$ versus execution time $H$ is reported for all the subjects, as well as their mean values.

It can be observed that, for the impedance control with constant parameters, the use of DC index (DCI) ensures better performance than the use of manipulability index (Man) both in terms of execution time and error on the path. This is true also for variable impedance control even though the use of variable parameters reduces the error on the path in spite of the strategy used to solve the redundancy.

Last but not least, all the subjects involved in the experiments have confirmed that the "feeling" of the manual guidance (in terms of intuitiveness and response of the 


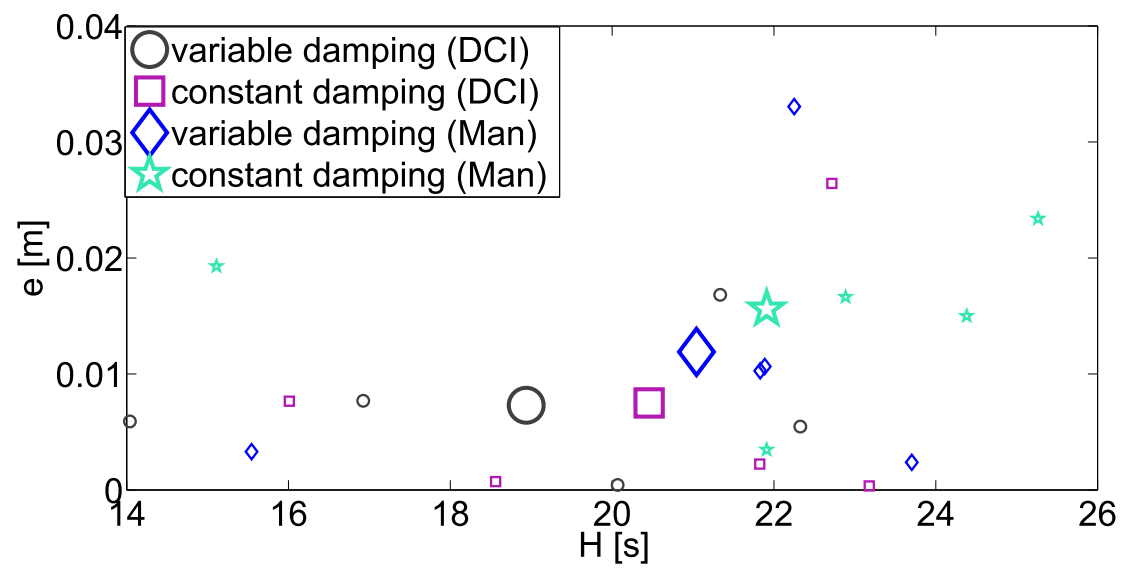

Figure 9

Values of the length error $e$ and execution time $H$ in the experiments on five subjects using variable and low constant impedance; both manipulability index and DCI optimisation are used as secondary tasks. The bigger markers are the mean values on the five different subjects.

robot) improves when the DC index is adopted, i.e., when redundancy is used to decouple the natural end effector dynamics along the principal directions of the task.

\subsection{Impedance Modulation}

In order to accommodate the human movement during physical interaction, high impedance parameters are desired when the operator performs fine movements at low velocity while lower values of the parameters should be used for large movements at high velocity $[9,10,8]$. Thus, using a variable impedance strategy it is possible to vary the damping and mass properties of the robot on-line according to human unpredictable behaviour. A crucial point is the interpretation of the human intention. Taking into account that human perception is mainly influenced by the damping parameter, while, for a given damping, the desired (virtual) mass is crucial for stability, different strategies to vary the impedance parameters have been tested and compared in [7].

Comparing the performance in terms of execution time and accuracy we finally choose to vary the damping according to the absolute value of the end effector Cartesian velocity and to set the mass as low as possible. Namely, when the velocity is high, the damping force is reduced, so that the operator can move the end effector with minimum effort and the execution time can be reduced; vice versa, at low velocity, the damping force is increased to improve accuracy. On the other hand, the virtual mass is set so as the parameters of the system remain in the stability region.

The relationships used to vary the damping for each of the Cartesian principal directions is

$D\left(x^{*}\right)=\min \left\{a \mathrm{e}^{-b\left|x^{*}\right|}, 5\right\}$, 
with $a=60$ and $b=4$. These parameters have been chosen in order to have a variation of the damping within the interval $[5,60] \mathrm{Ns} / \mathrm{m}$ for the possible range of velocities in the considered task. A saturation to the minimum value of $5 \mathrm{Ns} / \mathrm{m}$ is introduced in case of high velocity.

The variable impedance control has been compared with two different sets of constant impedance gains (chosen along the curve), namely: high damping $(\Lambda=1.1 \mathrm{~kg}$, $D=60 \mathrm{Ns} / \mathrm{m})$ and low damping $(\Lambda=1.1 \mathrm{~kg}, D=20 \mathrm{Ns} / \mathrm{m})$.

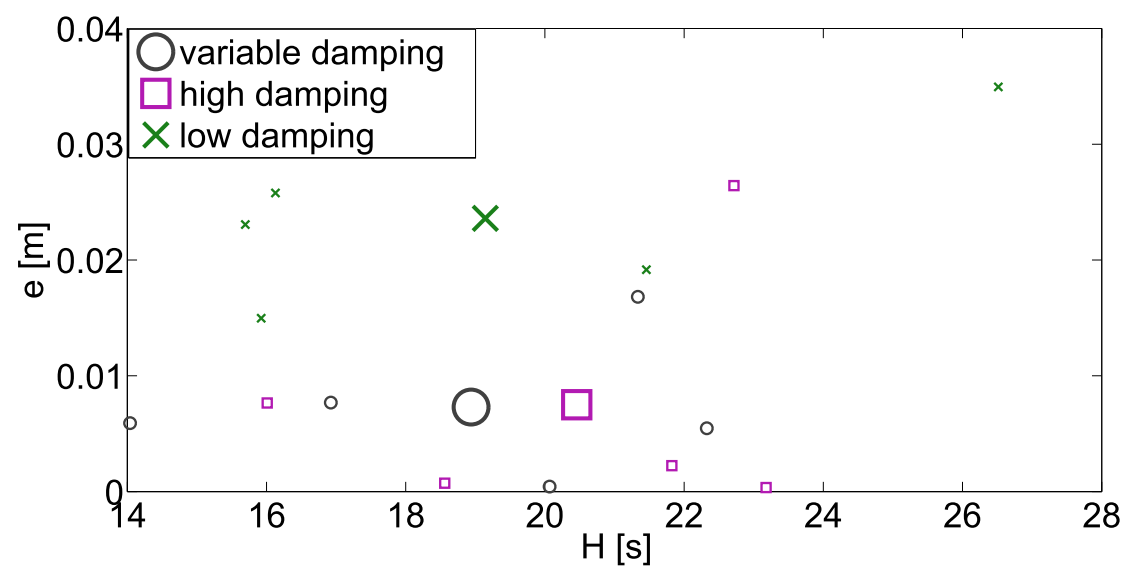

Figure 10

Values of length error $e$ and execution time $H$ in the experiments on five subjects using DCI optimisation, with the variable impedance control L1 and two different sets of constant parameters. The bigger markers are the mean values on the five different subjects.

The results, carried out on five different subjects, are shown in Fig. 10, where the execution time $H$ and the error on the length of the path are reported.

The constant impedance with high damping ensures higher accuracy with respect to the constant impedance with low damping, as expected. This result, however, comes at the expenses of the execution time and of the operator effort requested for the manual guidance.

Indeed, from Fig. 11 it can be verified that higher damping requires higher forces to be exerted to the end effector. On the contrary, impedance with low damping allows the task to be performed more easily, with less effort and time, but with less accuracy.

In conclusion, the variable impedance guarantees the best compromise between accuracy, execution time and effort of the operator (Fig. 10 and Fig. 11).

\section{Conclusions}

The use of impedance control both on Cartesian or task variables and in the null space of the main task was considered in this work. The redundancy of the system was utilised to ensure safe and dependable physical interaction, as well as to enhance intuitiveness and stability of the physical human-robot interaction. In the case of co-manipulation tasks, the benefit of impedance parameters modulation was 


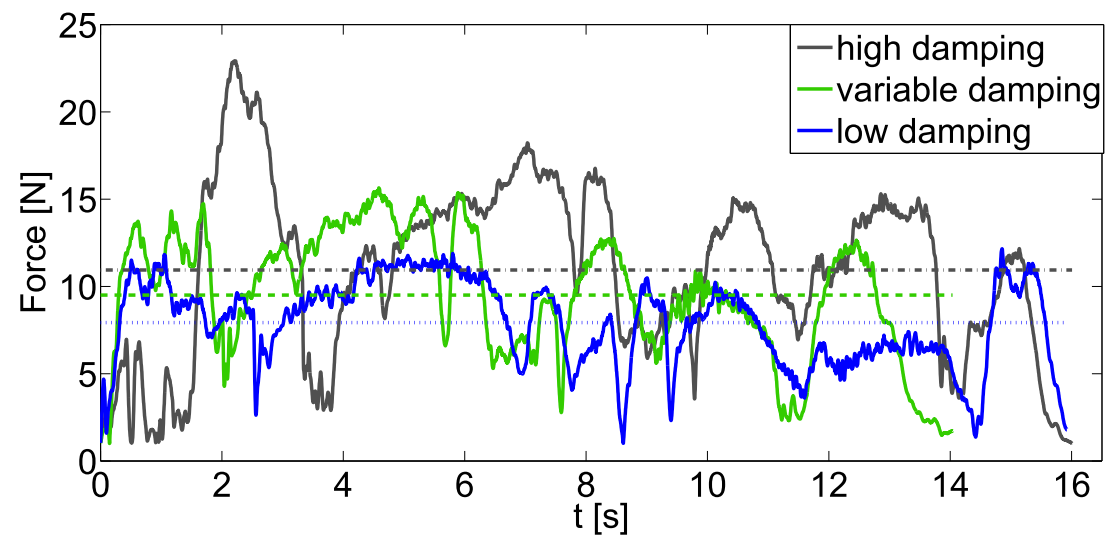

Figure 11

Norm and mean value of the contact forces for high, variable and low damping, for one subject.

investigated. The performance of the proposed algorithms has been experimentally tested on a torque controlled KUKA LWR4 robot.

\section{Acknowledgement}

This research has been partially funded by the EC Seventh Framework Programme (FP7) within SAPHARI project 287513 and RoDyMan project 320992.

\section{References}

[1] N. Hogan, "Impedance control: An approach to manipulation: Part i theory; Part ii implementation; Part iii applications," J. Dyn. Sys., Meas., Control, vol. 107, no. 12, pp. 1-24, 1985.

[2] H. Sadeghian, L. Villani, M. Keshmiri, and B. Siciliano, "Task-Space Control of Robot Manipulators with Null-Space Compliance," IEEE Transactions on Robotics, vol. 30, no. 2, pp. 493-506, 2014.

[3] F. Ficuciello, L. Villani, and B. Siciliano, "Variable Impedance Control of Redundant Manipulators for Intuitive Human-Robot Physical Interaction," IEEE Transactions on Robotics, vol. 31, no. 12, pp.850-863, 2015.

[4] A. D. Luca, A. Albu-Schäffer, S. Haddadin, and G. Hirzinger, "Collision detection and safe reaction with the DLR-III lightweight robot arm," in IEEE/RSJ Int. Conf. on Intelligent Robots and Systems, Beijing, China, 2006, pp. 1623-1630.

[5] O. Khatib, "A unified approach for motion and force control of robot manipulators: The operational space formulation," IEEE Journal of Robotics and Automation, vol. 3, no. 1, pp. 1115-1120, 1987.

[6] F. Ficuciello, H. Sadeghian, L. Villani, and M. Keshmiri, "Global Impedance Control of Dual-Arm Manipulation for safe Human-Robot Interaction," 10th IFAC Symposium on Robot Control, Dubrovnik, Croatia, 2012, pp. 767-773. 
[7] F. Ficuciello, A. Romano, L. Villani, and B. Siciliano, "Cartesian impedance control of redundant manipulators for human-robot co-manipulation," in IEEE/RSJ Int. Conf. on Intelligent Robots and Systems, Chicago, USA, 2014, pp. 2120-2125.

[8] R. Ikeura, T. Moriguchi, and K. Mizutani, "Optimal variable impedance control for a robot and its application to lifting an object with a human," in IEEE Int. Workshop on Robot and Human Interactive Communication, Berlin, Germany, 2002, pp. 500-505.

[9] A. Lecours, B. Mayer-St-Onge, and C. Gosselin, "Variable admittance control of a four-degree-of-freedom intelligent assist device," in IEEE Int. Conf. on Robotics and Automation, Saint Paul, Minnesota, USA, 2012, pp. 39033908 .

[10] V. Duchaine, B. Mayer-St-Onge, D. Gao, and C. Gosselin, "Stable and intuitive control of an intelligent assist device," IEEE Transactions on Haptics, vol. 5, no. 2, pp. 1939-1412, 2012.

[11] T. Insperger, L.L. Kovacs, P. Galambos, and G. Stepan, "Increasing the accuracy of digital force control process using the act-and-wait concept," IEEE Transactions on Mechatronics, vol. 15, no. 2, pp. 291-298, 2010.

[12] S. Buerger and N. Hogan, "Complementary stability and loop shaping for improved human-robot interaction," IEEE Transactions on Robotics, vol. 23, no. 2, pp. 232-244, 2007.

[13] O. Ma and J. Angeles, "The concept of dynamic isotropy and its applications to inverse kinematics and trajectory planning," in IEEE Int. Conf. on Robotics and Automation, San Francisco, CA, 1990, pp. 10-15. 